\title{
Article
}

\section{Liposome Mediated-CYP1A1 Gene Silencing Nanomedicine Prepared Using Lipid Film-Coated Proliposomes as a Potential Treatment Strategy of Lung Cancer}

Zhang, Mengtian, Wang, Qin, Wan, Ka-Wai, Ahmed, Waqar, Phoenix, David A, Zhang, Zhirong, Elrayess, Mohamed A, Elhissi, Abdelbary and Sun, Xun

Available at http://clok.uclan.ac.uk/28588/

Zhang, Mengtian, Wang, Qin, Wan, Ka-Wai, Ahmed, Waqar, Phoenix, David A, Zhang, Zhirong, Elrayess, Mohamed A, Elhissi, Abdelbary and Sun, Xun (2019) Liposome Mediated-CYP1A1 Gene Silencing Nanomedicine Prepared Using Lipid Film-Coated Proliposomes as a Potential Treatment Strategy of Lung Cancer. International Journal of Pharmaceutics, 566 . pp. 185-193. ISSN 03785173

It is advisable to refer to the publisher's version if you intend to cite from the work. http://dx.doi.org/10.1016/j.ijpharm.2019.04.078

For more information about UCLan's research in this area go to http://www.uclan.ac.uk/researchgroups/ and search for <name of research Group>.

For information about Research generally at UCLan please go to http://www.uclan.ac.uk/research/

All outputs in CLoK are protected by Intellectual Property Rights law, including Copyright law. Copyright, IPR and Moral Rights for the works on this site are retained by the individual authors and/or other copyright owners. Terms and conditions for use of this material are defined in the policies page. 


\section{Accepted Manuscript}

Liposome Mediated-CYP1A1 Gene Silencing Nanomedicine Prepared Using Lipid Film-Coated Proliposomes as a Potential Treatment Strategy of Lung Cancer

Mengtian Zhang, Qin Wang, Ka-Wai Wan, Waqar Ahmed, David A Phoenix, Zhirong Zhang, Mohamed A. Elrayess, Abdelbary Elhissi, Xun Sun

PII: S0378-5173(19)30349-7

DOI: https://doi.org/10.1016/j.ijpharm.2019.04.078

Reference: IJP 18326

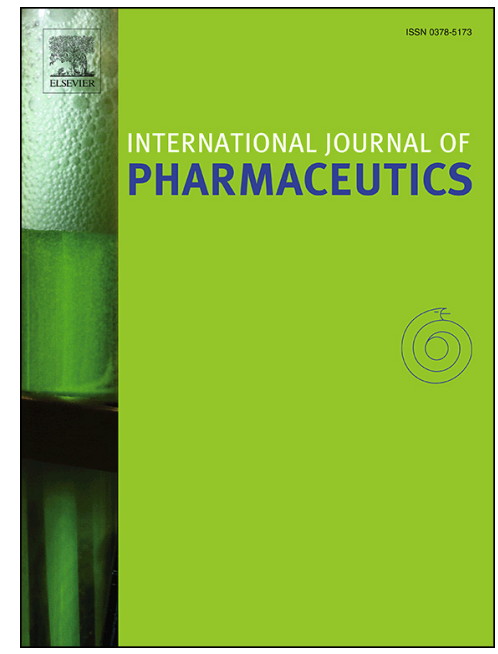

To appear in: International Journal of Pharmaceutics

Received Date: 19 November 2018

Revised Date: 17 April 2019

Accepted Date: 29 April 2019

Please cite this article as: M. Zhang, Q. Wang, K-W. Wan, W. Ahmed, D.A. Phoenix, Z. Zhang, M.A. Elrayess, A. Elhissi, X. Sun, Liposome Mediated-CYP1A1 Gene Silencing Nanomedicine Prepared Using Lipid Film-Coated Proliposomes as a Potential Treatment Strategy of Lung Cancer, International Journal of Pharmaceutics (2019), doi: https://doi.org/10.1016/j.ijpharm.2019.04.078

This is a PDF file of an unedited manuscript that has been accepted for publication. As a service to our customers we are providing this early version of the manuscript. The manuscript will undergo copyediting, typesetting, and review of the resulting proof before it is published in its final form. Please note that during the production process errors may be discovered which could affect the content, and all legal disclaimers that apply to the journal pertain. 
Liposome Mediated-CYP1A1 Gene Silencing Nanomedicine Prepared

Using Lipid Film-Coated Proliposomes as a Potential Treatment Strategy

Mengtian Zhang ${ }^{1}$, Qin Wang ${ }^{1}$, Ka-Wai Wan' ${ }^{2}$,Waqar Ahmed ${ }^{3}$, David A Phoenix 4 , Zhirong

\section{*Corresponding author 1}

18 Dr. Abdelbary Elhissi

19 Office of Vice President (Research and Graduate Studies)

20 Qatar University, Doha, Qatar \&

21 College of Pharmacy, Qatar University

22 Doha, Qatar, P.O. Box 2713

23 Tel: +974 44035632

24 E-mail: aelhissi@qu.edu.qa

28 Prof. Xun Sun 
West China School of Pharmacy

30 Sichuan University, No. 17, Section 3, Southern Renmin Road

31 Chengdu 610041, People's Republic of China

32 Tel.: +8628 85502307

33 Fax: +862885501615

34 E-mail: xunsun22@gmail.com

35

36

37

38

39

40

41

42

43

44

45

46

47

48

49

50

51

52

53

54

55

56

57

58

59

60

61

62

63 


\section{ABSTRACT:}

66 The occurrence of lung cancer is linked with tobacco smoking, mainly through the

67 generation of polycyclic aromatic hydrocarbons (PAHs). Elevated activity of cytochrome

68 P4501A1 (CYP1A1) plays an important role in the metabolic processing of PAHs and its

69 carcinogenicity. The present work aimed to investigate the role of CYP1A1 gene in PAH-

70 mediated growth and tumor development in vitro and using an in vivo animal model. RNAi

71 strategy was utilized to inhibit the overexpression of CYP1A1 gene using cationic

72 liposomes generated using a lipid film-coated proliposome microparticles. Treatment of

73 PAH-induced human alveolar adenocarcinoma cell line with cationic liposomes carrying

74 CYP1A1 siRNA resulted in down regulation of CYP1A1 mRNA, protein as well as its

75 enzymatic activity, triggering apoptosis and inhibiting multicellular tumor spheroids

76 formation in vitro. Furthermore, silencing of CYP1A1 gene in BALB/c nude xenografts

77 inhibited tumor growth via down regulation of CYP1A1 expression. Altogether, our

78 findings showed that liposome-based gene delivery technology is a viable and stable

79 approach for targeting cancer causing genes such as CY1PA1. This technology facilitated

80 by the use of sugar particles coated with lipid films has demonstrated ability to generate

81 anticancer effects that might be used in the future for therapeutic intervention and treatment

82 of lung cancer. 
83 KEYWORDS: Apoptosis, Cancer, CYP1A1, Lung, siRNA, Smoking, Tobacco

\section{1. INTRODUCTION}

85 Lung cancer has become a leading cause of death worldwide due to the increased

86 environmental contamination with inhalable carcinogens occurring as byproducts of

87 combustion processes and unhealthy habits such as tobacco smoking (Field and Withers,

88 2012). Despite the efforts made to improve the life quality of cancer patients, a proper

89 understanding of the pathogenesis of lung cancer is still missing, resulting in poor treatment

90 outcomes and severe adverse effects of chemotherapy and radiotherapy (Brambilla and

91 Gazdar, 2009). Susceptibility of lung to carcinogenesis is based on the metabolic imbalance

92 between induction and detoxification pathways, with a significant role of external inducing

93 factors (Hecht, 1999).

94 Polycyclic aromatic hydrocarbons (PAHs) produced by tobacco smoking are involved in

95 the activation and development of lung cancer (Armstrong et al., 2004; Hecht, 1999).

96 Although the detailed mechanism of how this group of carcinogens disrupts the

97 homeostasis of lung cells is still unclear, studies have concluded that PAHs can induce the

98 overexpression of cytochrome P4501A1 gene (CYP1A1), an important member of a large

99 family of cytochrome P450 enzymes involved in the metabolism of PAHs (Shimada and

100 Fujii-Kuriyama, 2004). Consequently, many highly electrophilic metabolic intermediates 
101 can be produced, causing irreversible damage to human tissues and inducing cancer

102 occurrence (Shimada and Fujii-Kuriyama, 2004). Therefore, targeting of CYP1A1 gene

103 may be a promising therapeutic strategy especially for smoking-related lung cancer

104 (Androutsopoulos et al., 2009; Bruno and Njar, 2007). The induction of CYP1A1 primarily

105 occurs when the inducer binds to the ligand-activated transcriptional factor aryl

106 hydrocarbon receptor (AhR) (Guigal et al., 2000). Flavonoid galangin, an antagonist

107 against AhR, has been considered as an inhibitor candidate to decrease the CYP1A1

108 expression (Ciolino and Yeh, 1999). However, multi-targeted properties of this drug may

109 lead to non-specific inhibition of the other members of the P450 gene family (Murakami et

110 al., 2008; Sak and Everaus, 2015), suggesting a better and specific strategy is needed to

111 target CYP1A1 gene for therapeutic intervention and treatment of lung cancer.

112 RNA interference (RNAi) is a gene silencing technology at the transcriptional level and

113 works through specifically targeting mRNA via sequence-specific matches, resulting in

114 degradation of the target mRNA (Agrawal et al., 2003). siRNA technology promises

115 greater advantages over conventional drugs currently in the market for its high targeting

116 selectivity and low toxicity; however, pharmacokinetic properties of siRNA are

117 unpredictable and its cellular uptake is poor (Lorenzer et al., 2015). Accordingly, specific 
118 siRNA-mediated silencing of CYP1A1 expression with improved kinetics and uptake by

119 target cells is urgently warranted.

120 As widely used vehicles in nucleic acid delivery, non-viral vectors such as cationic

121 liposomes are much safer than viral vectors (Khurana et al., 2013). Furthermore, compared

122 to polymeric vectors, cationic liposomes may offer higher transfection and greater

123 biocompatibility (Ruozi et al., 2003). Novel cationic lipids conjugated with functional

124 targeting groups may offer a great potential for use in the preparation of cationic liposomes

125 ( Ruozi et al., 2003; Kim et al., 2010b; Sun et al., 2018).

126 Liposomes manufactured using the traditional thin-film hydration technique with

127 subsequent prepartion as liquid dispersions are unstable during storage owing to the

128 liability of phospholipids to hydrolysis and oxidation, with subsequent compromise of the

129 validity of liposomes as drug carriers (Grit and Crommelin, 1993). Alternatively,

130 proliposomes are stable powdered phospholipid formulations prepared by coating

131 carbohydrate carrier particles with thin phospholipid films using modified rotary

132 evaporators (Elhissi et al., 2006; Gala et al., 2015). Liposomes can be generated from

133 proliposomes via the addition of aqueous phase and shaking (Elhissi et al., 2006; Gala et

134 al., 2015). Several reports have established the suitability of manufacturing thin-film-based

135 proliposome powders on a large scale, for instance by using fluidized-bed coating (Chen

136 and Alli, 1987; Kumar et al., 2001; Gala et al., 2015). Liposomes generated from lipid film 
137 coated sugars (i.e. proliposomes) have been widely investigated for drug delivery. For

138 example, early reports have shown that oral delivery of non-steroidal anti-inflammatory

139 drugs in liposomes generated from proliposomes can protect against gastric ulceration in

140 experimental animals (Katare et al., 1990). Proliposomes have also been investigated for

141 nasal delivery of propranolol hydrochloride and nicotine (Ahn et al., 1995; Jung et al.,

142 2000a), and for parenteral administration of antifungal drugs (e.g. amphotericin B) (Payne

143 et al., 1987), and anticancer agents such as methotrexate (Park et al., 1994) and doxorubicin

144 (Wang et al., 2000), and for transdermal delivery of nicotine (Hwang et al., 1997; Jung et

145 al., 2000b). We have previously shown that proliposomes made by coating sucrose with

146 lipid films can generate inhalable liposomes when hydrated in situ within medical

147 nebulizers (Elhissi et al., 2012). More recent investigators have shown that diltiazem HCL

148 liposomes generated from proliposomes could be used for topical treatment of glioma

149 (Mokhtar Ibrahim et al., 2013) and dermatitis (Jahn et al., 2014) using animal models.

150 Proliposomes made by film-ciating of sugar particles have recently been demonstrated to be

151 compressible into tablets, with properties being dependent on formulation (Khan et al., 152 2018).

154 In this study, lipid film-based proliposome technology was employed for the preparation of 155 cationic liposomes-siRNA (CL-siRNA) formulations for targeting the CYP1A1 gene. AhR- 
156 mediated induced expression of CYP1A1 in A549 adenocarcinoma cell line was used to

157 model smoking induction of CYP1A1 expression. The effects of CYP1A1 silencing with

158 CYP1A1 CL-siRNA on CYP1A1 expression, CYP1A1enzyme activity, cell apoptosis and

159 tumor spheroids formation were verified in induced A549 cell lines. The effect of CYP1A1

160 silencing on tumor regression was further investigated in the induced A549 tumors in

161 xenograft BALB/c-nude mice.

163 2. MATERIALS AND METHODS

164

165 2.1. Materials

166 1,2-dioleoyl-3-trimethylammonium-propane

(DOTAP) and dioleoyl-

167 phosphatidylethanolamine (DOPE) were purchased from Avanti Polar-Lipids Inc.

168 (Alabaster, AL, USA). Cholesterol was obtained from Biotech Co. Ltd (Shanghai, China),

169 and 3-methylcholanthrene (3-MC) was purchased from SUPELCO Co.

170 (Pennsylvania ,USA). Human CYP1A1 siRNA was chemically synthesized and purified

171 via HPLC by RiboBio (Guangzhou, China). Goldview staining was purchased from

172 Guangzhou Geneshun Biotech Ltd (Shanghai, China). RNA prep pure cell kit was

173 purchased from TIANGEN (Beijing, China). The sequence for siRNA was as follows:

174 siRNA against CYP1A1: sense, 5'-GGCCUGAAGAAUCCACCAG-3'; antisense, 3'- 
175 CUGGUGGAUUCUUCAGGCC-5'. FAM-siRNA and the same sequence was obtained

176 from Sangon Biotech (Shanghai, China). Lipofectamine2000 was obtained from Invitrogen 177 (USA).

178 2.2. Preparation of liposome-siRNA complexes using lipid-coated particulate-based 179 proliposomes

180 Sorbitol particles $(300-500 \mu \mathrm{m})$ were placed in $50 \mathrm{ml}$ pear-shaped flask and attached to a

181 modified rotary evaporator with a feed-line tube. The flask was partially immersed in a

182 water bath $\left(37^{\circ} \mathrm{C}\right)$. A chloroform solution containing DOTAP, DOPE and Cholesterol

183 (3:4:3 mole ratio) was injected in portions $(0.5 \mathrm{~mL}$ each) via the feed-line using a syringe

184 and by releasing the vacuum for a few seconds using a valve fitted on top of the condenser

185 to allow lipid solution to be drawn through the feed-line and be sprayed onto the sorbitol

186 carrier particles. After each addition, complete evaporation of chloroform was allowed

187 before injecting the next portion. After solvent was completely evaporated, the solid

188 particles of proliposomes were collected and stored in glass vials in the freezer $\left(-18^{\circ} \mathrm{C}\right)$.

189 Proliposomes were hydrated with water to form liposomes $(1 \mathrm{mg} / \mathrm{mL})$ followed by probe-

190 sonication. The sonicated cationic liposomes (CL) were mixed with siRNA in RNase-free

191 water using vortex-mixing and incubated for $30 \mathrm{~min}$ at room temperature to form CL-

192 siRNA complexes.

193 
194 2.3. Scanning electron microscopy (SEM) of lipid film coated proliposomes

195 Microparticles made by coating sorbitol carrier with lipid film were positioned onto a 196 carbon pad (Agar Scientific, UK), and coated with a thin film of gold using the sputter 197 coater of the microscope (Bio-Rad, England). The morphology of the resultant 198 microparticles was investigated under vacuum using the Quanta 200 scanning electronic 199 microscope.

\subsection{Size analysis and zeta potential studies of CL-siRNA}

202 Size analysis and zeta-potential studies of CL and CL-siRNA complexes were conducted 203 using Photon Correlation Spectroscopy (PCS) and laser Doppler velocimetry, respectively.

204 The studies were performed using the Malvern ZetaSizer Nano ZS90 (Malvern Instruments 205 Ltd, UK) upon selecting the right software for each type of analysis. Size and size 206 distribution were expressed by the instrument as the mean hydrodynamic diameter and 207 polydispersity index (PDI), respectively.

210 Human alveolar adenocarcinoma, A549 lung cancer cell line was obtained from American

211 Type Culture Collection (Rockville, MD, USA). A549 cell line was cultured in RPMI 1640

212 medium (HyClone, USA) supplemented with 10\% fetal bovine serum (Minhai, China), 100 
$213 \mathrm{U} / \mathrm{ml}$ penicillin, $100 \mathrm{mg} / \mathrm{ml}$ streptomycin. Cell culture was performed in an incubator

214 maintained at $37^{\circ} \mathrm{C}$ in a humidified environment containing $5 \% \mathrm{CO}_{2}$.

217 2.6. Agarose gel retardation assay

218 To confirm formation of the complexes, the agarose gel retardation assay was employed to

219 select the optimal charge ratio between cationic liposomes and negatively charged siRNA.

220 CL-siRNA complexes were prepared at various molar ratios, and then run through a $2 \%$

221 agarose gel. The mobility of siRNA complexed with cationic liposomes was visualized by

222 GoldView staining.

\section{2.7. RNase protection assay of siRNA in cationic liposome complexes}

225 An ideal siRNA delivery system is expected to protect siRNA against RNase enzymatic

226 degradation. In order to monitor siRNA degradation by nuclease, firstly, siRNA-CL

227 complexes were prepared at a final siRNA concentration of $5 \mu \mathrm{M}$ and then incubated in the

228 presence of $0.1 \mathrm{mg} / \mathrm{ml}$ RNase for $30 \mathrm{~min}$ at $37^{\circ} \mathrm{C}$. An aliquot $(20 \mu \mathrm{l})$ was removed and snap-

229 frozen at $-80^{\circ} \mathrm{C}$ at every time point. All samples were thawed on ice when they were

230 collected and immediately mixed with $5 \mu \mathrm{l}$ of a $100 \mathrm{mM}$ Triton X100 solution and $25 \mu \mathrm{l}$ of

231 RNA-extraction mixture (phenol/chloroform/isoamyl alcohol; 25:24:1). The siRNA was 
232 precipitated with ethanol, electrophoresis was performed on agarose gel (2\%) and

233 visualization took place by GoldView staining (Buyens et al., 2008).

\subsection{Cell model with high expression of CYP1A1}

237 For the purpose of simulating the gene induction pathway to obtain a cell model with high

238 CYP1A1 gene expression, an induction assay was carried out on A549 cells which are

239 common for CYP1A1 gene research (Fazili et al., 2010). Cells were seeded in 12-well

240 plates at a density of $1 \times 10^{4}$ cells per well, followed by $24 \mathrm{~h}$ incubation at $37^{\circ} \mathrm{C}$ in a

241 humidified environment containing $5 \% \mathrm{CO}_{2}$, and cells were treated with 3-MC with a final

242 concentration of $5 \mu \mathrm{M}$ for further $24 \mathrm{~h}$ (3-MC was dissolved in DMSO). 3-MC is one of the

243 most potent PAH carcinogens, which is usually used in the induction of CYP1A1 via the

244 AhR mechanism (Abdelrahim et al., 2003). After the induction, the induced cells were

245 collected and used in the subsequent experiments.

247 2.9. Cellular uptake of siRNA in induced A549 lung cancer Cells

248 Transfection of FAM-siRNA (what is FAM-siRNA) was performed in induced A549 cells.

249 The induced A549 cells were seeded at a concentration of $5 \times 10^{5}$ cells per well in six-well

250 plates. The cells were grown to a confluency between $60 \%$ and $80 \%$ and washed with pre- 
251 warmed $\left(37^{\circ} \mathrm{C}\right) \mathrm{PBS}$, and then they were incubated with $100 \mathrm{nM}$ liposome-free FAM-

252 siRNA or $100 \mathrm{nM}$ FAM-siRNA-liposome complexes in serum-free medium. Following

253 incubation for $4 \mathrm{~h}$, the medium was replaced and the cells were washed with PBS twice,

254 and then analyzed using flow cytometry (Beckman Coulter, USA) and examined under a

255 fluorescence microscope. siRNA complexed with Lipofectamine2000 (Lipo2000) was used

256 as a positive control in the experiments.

\subsection{Silencing of 3-MC induced CYP1A1 gene in A549 lung cancer cell line}

259 For evaluation of the mRNA of CYP1A1 gene in vitro and in vivo, RNA was extracted

260 from cells $24 \mathrm{~h}$ after transfection with CYP1A1-specific siRNA $(\mathrm{n}=3)$ or from A549 lung

261 tumor $(n=3)$, respectively, using RNA prep Pure cell kit. cDNA was then obtained by

262 reverse transcription of the total RNA using the TIANscript RT kit and the CYP1A1 (sense,

263 5'-GGCCUGAAGAAUCCACCAG-3'; antisense, 3'-CUGGUGGAUUCUUCAGGCC-5').

264 mRNA levels were analyzed using the SosoFast ${ }^{\mathrm{TM}}$ EvaGreen Supermix on iCycler iQ ${ }^{\mathrm{TM}} 5$

265 system (Bio-Rad, USA) and $\beta$-actin was used as internal control. The PCR reaction was

266 conducted at $95^{\circ} \mathrm{C}$ for 3 min followed by 40 cycles of $95^{\circ} \mathrm{C}$ for $5 \mathrm{~s}$, and $56^{\circ} \mathrm{C}$ for $10 \mathrm{~s}$ in the

267 iQ ${ }^{\mathrm{TM}} 5$ Real-Time PCR Detection System. The expression of CYP1A1 was analyzed and

268 normalized using the $2^{\Delta \mathrm{Ct}}$ method relative to the expression of $\beta$-actin. 


\subsection{CYP1A1 enzyme assays}

271 To further study the silencing effect of siRNA on CYP1A1, the enzyme activity as well as

272 the content of CYP1A1 was assessed. The CYP1A1 enzyme activity was determined by

273 Human CYP1A1 fluorescence quantitative detection kits (Genmed Scientifics INC.USA).

274 The CYP1A1 enzyme content was measured using Human CYP1A1 ELISA kits (R\&D

275 systems, USA). Both assays were performed following the relevant suppliers' instructions.

$277 \quad$ 2.12. Apoptotic assays

278 To examine the interactions between CYP1A1 gene regulation and the induced growth of

279 tumor cells, different groups were designed in the cell apoptosis experiment. Induced A549

280 cells were treated with CYP1A1-specific siRNA (100 nM) or complexed with cationic

281 liposome in serum-free medium for $4 \mathrm{~h}$ and then further incubated in fresh completed

282 medium. Cells were washed with PBS and digested in trypsin for suspension after

283 incubation for $48 \mathrm{~h}$, followed by double staining with FITC-Annexin $\mathrm{V}$ and propidium

284 iodide using the cell apoptotic analysis kit (Beyotime, China) following the manufacturer's

285 instructions. Flow cytometry was used for investigation of cell apoptosis $(\mathrm{n}=3)$. Further

286 studies were performed to investigate the apoptosis mechanism. Caspases are the critical

287 proteins responsible for apoptosis. These proteins are classified as initiators or executioners

288 depending on their point of entry into the apoptotic cascade. It has been confirmed that 
289 there were two main apoptosis pathways mediated by caspases (Boatright and Salvesen,

290 2003). Among all the family members in this pathway, caspase 3 was considered as the

291 final executioner, and meanwhile, caspase 8 and caspase 9 are the key initiator proteins

292 which exist in the extrinsic and intrinsic apoptotic pathways, respectively. The three

293 caspases were firstly detected with Caspase Activity Assay Kits (Beyotime, China).

\section{2.13. Multicellular tumor spheroids (MCTSs) assays}

296 Multicellular tumor spheroids (MCTSs) may provide an appropriate model to identify the

297 drug effect in vitro for its similarity to the tumor formation in vivo (Friedrich et al., 2009).

298 A549 cells were cultured in a modified tumor sphere medium. The medium is comprised of

299 recombinant fibroblast growth factor (EGF) $(10 \mathrm{ng} / \mathrm{ml})$, basic fibroblast growth factor

300 (bFGF) $(10 \mathrm{ng} / \mathrm{ml})$ and insulin $(4 \mathrm{U} / \mathrm{L})$, and plated at a density of $2 \times 10^{3}$ cells per well in 6-

301 well plates. Spheres were formed after 8-10 days incubation. After $24 \mathrm{~h}$ 3-MC induction,

302 spheres were treated with different groups of siRNA which were described in the gene

303 silence study at a siRNA final dose of $100 \mathrm{nM}$ for $4 \mathrm{~h}$. After further $72 \mathrm{~h}$ incubation, the

304 results were observed by microscope. 
308 The animal study protocol was approved by Institutional Animal Care and Use Committee

309 of the Sichuan University in China. Male BALB/c nude mice (weighing 20-23 g) were used

310 to investigate the antitumor efficacy of targeting CYP1A1 gene in vivo. Briefly, $1 \times 10^{7}$

311 A549 cells were re-suspended in $200 \mu \mathrm{l}$ serum-free RPMI 1640 medium and injected

312 subcutaneously into the right flank of the nude mice. After 5 weeks tumor-bearing mice

313 were randomly divided into four treatment groups (5 animals each). At days 1, 4, 7, 10, 13

314 and 16, mice were intratumorally injected with $100 \mu 1$ 10\% 3-MC solution. Then at days 2 ,

$3155,8,11,14$ and 17, mice were intratumorally injected again but with PBS, free siRNA or

316 CL-siRNA. Every treatment was based on the dose of $40 \mu \mathrm{g}$ siRNA per mouse. Calipers

317 were used in this work to measure the tumor progression of every mouse. Tumor volumes

318 were calculated as length $\times$ width $\times$ width $\times 0.5\left(\mathrm{~mm}^{3}\right)$. At the day 18 , three animals from each

319 group including control were sacrificed, and the tumors were excised. The measurements of

320 CYP1A1 gene silencing effect were conducted as described earlier.

$322 \quad$ 2.15. Statistical analysis

323 Values were presented as mean $( \pm$ SD) unless otherwise stated. The differences between

324 groups were analyzed using the Student's t-tests and one-way analysis of variance

325 (ANOVA) with Bonferroni tests for multiple-group analysis. A probability level of $\mathrm{P}<$

3260.05 was considered to indicate significant difference between the groups. 


\section{RESULTS}

3.1. Physical characterization of proliposomes, cationic liposomes (CL) and CLsiRNA complexes

333 The surface morphology of proliposome powders prepared through coating sorbitol

334 particles with lipid film was examined by scanning electron microscopy (SEM) (Figure 1).

335 The high porosity of sorbitol (Figure 1a) facilitated coating of the lipid on the carrier

336 surfaces (Figure 1b). Our SEM observations using cationic lipids to coat sorbitol particles

337 is in concordance with the previous findings using neutral lipids such as

338 dimyristoylphosphatidylcholine coated onto sorbitol particles (Payne et al., 1986). Our

339 study also further confirms that sorbitol is a highly suitable carrier for coating with lipid

340 films and preparation of proliposomes because of its microporous structure. In another

341 study, we demonstrated that the film coating proliposome technology can be scaled up

342 using fluid-bed coating equipment that can deposit a lipid film on carbohydrate particles

343 (e.g. sucrose), generating liposomes that can successfully entrap conventional small

344 molecules, such as the antiasthma steroid beclometasone dipropionate (Gala et al., 2015). In 
345 the present investigation, through a smaller scale of manufacturing using a modified rotary

346 evaporator equipped with a feed tubeline, proliposomes made by coating sorbitol with

347 cationic lipids were prepared. Upon hydration (including or excluding siRNA) and probe-

348 sonication, cationic liposomes were generated. The measured size of the siRNA-free

349 vesicles was as small as $85 \pm 3.2 \mathrm{~nm}$ and the size distribution, expressed by PDI, was as low

350 as 0.165 . The uniform coating of sorbitol particles (Figure 1b) justifies the facilitated

351 generation of liposomes in the nano-size range and the narrow size distribution (i.e. low

352 PDI) (Figure 1d). Transmission electron microscopy (TEM) images confirmed the uniform

353 round shape of the gene-free cationic liposomes, which were also similar to those

354 incorporating siRNA, suggesting that the genetic material was complexed with the

355 liposomes, with no apparent formation of siRNA aggregates (Figure 1c). 1,2-dioleoyl-3-

356 trimethylammonium-propane (DOTAP) in the formulation conferred the liposomes with a

357 positive surface charge of about $+43 \mathrm{mV}$ (sorbitol solution $\mathrm{pH}=7.5$ ) (Figure 1d). For

358 formulations incorporating siRNA, the integrity of siRNA was studied (Figure 1e). Varying

359 charge ratios of CL to siRNA (N/P ratio) were prepared at fixed siRNA concentration (100

$360 \mathrm{nM}$ ). With the N/P ratio higher than 4, the migration of siRNA was completely retarded,

361 indicating good binding efficiency of CL with siRNA and successful formation of the

362 complexes (Figure 1e, f). On the other hand, size and zeta potential of CL-siRNA

363 complexes were $90 \mathrm{~nm}$ and $+30 \mathrm{mV}$, respectively at the N/P mole ratio of 4:1 (Figure 1d), 
364 which contributed to the good dispersion properties and stability of CL-siRNA complexes.

365 Considering all the results above, $\mathrm{N} / \mathrm{P}=4: 1$ was chosen as the optimal charge ratio for CL-

366 siRNA complex formation.

368 Agarose gel assay is an established technique for checking the formation of complexes

369 between liposomes and genetic materials (e.g. siRNA) (Kim et al., 2010a). To assess the

370 ability of liposomes to protect siRNA from degradation, the stability of siRNA in RNase

371 solution was tested. As shown in Figure 1f, free siRNA was completely degraded upon

372 exposure to RNase. By contrast, when siRNA was incorporated into cationic liposomes, the

373 genetic material was intact for at least $4 \mathrm{~h}$, indicating that liposomes have provided short-

374 term protection for siRNA against enzymatic degradation. In this study, we made powdered

375 cationic formulations of proliposomes by film coating the sorbitol sugar with cationic lipid.

376 This can readily generate liposomes complexing with siRNA via addition of aqueous phase

377 and sonication just on the day of administration; hence, storage instability of liposome

378 dispersions is avoided. cell line 
382 After incubation with 3-MC $(5 \mu \mathrm{M})$ for $24 \mathrm{~h}$, the induced A549 cells were collected to 383 investigate the target gene CYP1A1 expression level. All samples were analyzed by RT384 PCR, which suggested that the mRNA level of CYP1A1 in induced cells was about 7 times

385 higher than that in the normal cells. Enhanced CYP1A1 expression was maintained for at 386 least $48 \mathrm{~h}$ after single induction.

388 CL were compared with Lipo2000, a commonly used positive control for siRNA delivery,

389 for evaluation of the siRNA delivering ability. FAM-labeled siRNA was prepared alone or 390 mixed with CL or Lipo2000 at a final concentration of $100 \mathrm{nM}$. Both flow cytometry and 391 confocal microscopy were used to investigate the uptake efficiency of the liposomes in 392 A549 cells. The results indicated that both CL and Lipo2000 effectively delivered siRNA to 393 cells (Figure 2a), and significantly improved the uptake efficiency compared with free 394 siRNA solution (Figure 2b).

\subsection{Silencing of CYP1A1 gene expression in A569 lung cancer cell line}

397 Transfection of induced A549 cells with CL-CYP1A1-siRNA caused a 7-fold down398 regulation of CYP1A1 gene expression. Similar results were obtained with transfection 399 using CYP1A1-siRNA Lipo2000 control. On the other hand, free (i.e. naked) CYP1A1400 siRNA and negative control siRNA (NC siRNA) did not show any marked silencing effect 
401 on CYP1A1 gene expression (Figure 3a). All agents were tested in the induced cells, and 402 the unstimulated A549 cells were used as a negative control. The silencing effects of CL403 CYP1A1-siRNA on CYP1A1 protein levels and enzymatic activity was also seen (Figure $4043 \mathrm{~b}$ and 3c), confirming successful retardation of gene expression target. CL-siRNA 405 prepared using the film-coating proliposome technology caused a similar knockdown 406 efficiency compared to the positive control Lipo2000. This clearly demonstrates that the 407 facile approach of generating CL-siRNA using the proliposome technology was successful 408 at providing a more stable powdered formulation than conventional liposomes. It was also 409 capable of retarding the gene expression in levels similar to those of the established 410 Lipo2000 transfection reagent.

412 3.4. Knockdown of CYP1A1 gene induces apoptotic cell death in 3MC- treated A549 $413 \quad$ cells

414 The number of apoptotic cells was quantified by FITC-Annexin V and propidium iodide 415 (PI) double-staining. CL-siRNA triggered apoptosis in induced A549 cells (Fig.4a). 3-MC 416 induced cells without further treatment were used as the negative control in these 417 experiments in order to eliminate the inducer influence on the results. Findings revealed 418 that 3-MC induction had a little impact on the cellular growth, whereas the induced cells 
419 tended to undergo apoptosis with CYP1A1 silencing through the intrinsic apoptotic 420 pathway marked by elevated caspase 3 and caspase 9, but not caspase 8, activities (Figure 421 4B), also confirmed by direct immunostaining (data not shown).

\subsection{The Effect of CYP1A1 gene silencing on sphere formation in A549 lung cancer}

425 Sphere formation assay was performed to investigate the effect of CYP1A1 silencing on

426 formation of spheroid colonies in vitro. Untreated induced A549 cells successfully

427 produced spheroid colonies when cultured in a modified tumor sphere medium. On the

428 other hand, spheres treated with CYP1A1 siRNA delivered by cationic liposomes or

429 Lipo2000 formulation showed a suppressive effect on the formation of sphere colonies. The

430 other groups including those untreated and mock did not exhibit this effect (Figure 5).

3.6. Antitumor efficacy of gene silencing of CYP1A1 in tumor-bearing nude mice

\section{using particulate-based proliposome technology}

434 In order to investigate the impact of CYP1A1 silencing on tumor progression in vivo, we 435 determined the antitumor efficacy of CL-siRNA in A549 xenograft nude mice model

436 (Figure 6a). Results showed that growth rate of tumor with cationic liposome or Lipo2000 
437 was significantly slower than that observed in the control groups including animals injected 438 with PBS or naked (free) siRNA (Figure 6b/c). Moreover, the treatment caused down439 regulation of the expression of CYP1A1 gene in the tumors as detected by RT-PCR on the 440 third day after giving the intratumoral dose (Figure 6d). Thus, the reduction of CYP1A1 441 gene in induced A549 cells mediated by siRNA gave a significant tumor growth inhibition.

\section{4. DISCUSSION}

444 In this study we report that liposome-based gene delivery technology is a viable and stable 445 approach for targeting the cancer causing gene CY1PA1. A major issue for liposomes is 446 their instability as liquid dispersion, commonly when prepared using the thin-film 447 hydration technique (Grit and Crommelin, 1993). This was overcome in the present study 448 by using the film-coating proliposome technology to prepare powdered lipid formulations 449 that, when needed, can be used to generate CL-siRNA complexes.

450 This technology, as demonstrated in our study, can potentially be considered for therapeutic

451 intervention and treatment of lung cancer, one of the most common types of cancer and a 452 leading cause of death (Torre et al., 2015). This approach comes as part of ongoing efforts

453 to ameliorate the outcomes related to the undesirable pharmaceutical, pharmacokinetic and 454 pharmacodynamic properties of lung cancer drugs, such as solubility, toxicity, stability, and 
455 lack of selective effect on the cancerous cells (Tiwari et al., 2012). These properties can be 456 enhanced by using drug vectors that are highly biocompatible and biodegradable 457 (Zarogouldis et al., 2012).

458 Continuous exposure to tobacco smoking can induce the expression of CYP1A1, a gene

459 present in extra hepatic tissues (Androutsopoulos et al., 2009), that is involved in the 460 metabolic activation of PAH produced from tobacco smoking. After the induction, high 461 CYP1A1 gene expression can contribute to the carcinogenic derivatives production and 462 may initiate neoplastic transformation (Whitlock, 1999). Stimulated bronchial epithelial 463 cells express high levels of CYPT1A1 gene when induced by tobacco or environmental 464 pollutants, predisposing them to lung cancer (Mercer et al., 2006). Hence, A549 human 465 alveolar basal epithelial cell line represents a valuable model for the mechanistic studies 466 involving induction of the pulmonary CYP system (Giard et al., 1973). In this study, we 467 constructed a cell model on the basis of AhR mechanism through which CYP1A1 can be 468 activated to a high level using 3-MC as previously reported (Hukkanen et al., 2000). In the 469 induced cells, a high CYP1A1 gene expression was observed, similar to that seen in 470 cancerous cells exposed to air contaminants. In our study, the 3-MC concentration was 471 optimized to exhibit low toxicity and relatively high induction efficiency. 
472 Limited studies have reported the relationship between inhibition of CYP1A1 gene and

473 lung cancer therapy (Androutsopoulos et al., 2009). Flavonoid (such as quercetin), for 474 example, was previously reported to inhibit CYP1A1 induction (Ciolino and Yeh, 1999). In 475 the present work, we used RNAi as the inhibition strategy in lung cancer cells. Successful 476 therapy using siRNA depends on effective delivery and protection against RNase. Owing to 477 its large molecular weight and anionic nature, the uptake of siRNA by cancer cells is very 478 poor, making the use of appropriate delivery systems highly advantageous (Gala et al., 479 2015). To overcome these issues, we prepared cationic liposomes via the lipid-coating 480 proliposome technology shown previously to be suitable for large scale production (Gala et 481 al., 2015). Using fluidized bed coating, the solid proliposomes produced can be stored at $48218^{\circ} \mathrm{C}$ until needed for subsequent generation of liposomes, providing stability for several 483 months (data not shown). In addition mass production and storage stability of proliposomes 484 (as liposome precursors), the cationic liposomes were able to protect siRNA from nucleases 485 and facilitated efficient transportation of siRNA into the cytoplasm, resulting in gene 486 silencing effects similar to those exhibited by the commercially established Lipo2000. 487 Indeed, both our in vitro and in vivo results indicated that CYP1A1 gene silencing by 488 siRNA can regulate the cancer in the induced cells. Our data showed that the down489 regulation of $C Y P 1 A 1$ gene induced cellular apoptosis and interfered with the formation of 490 tumor spheres in vitro and inhibited tumor development in BALB/c nude xenograft model. 
491 Various murine models were established for the evaluation of novel therapeutics and 492 examination of the molecular mechanisms underlying transformation, invasion and 493 metastasis (Kellar et al., 2015). The A549 xenograft model was chosen in this study for the 494 convenience of tumor measurement by making the cancer cells readily accessible (Kellar et 495 al., 2015). Therefore in vivo results remain preliminary in nature and inconclusive.

496 However, the emerging data confirm the validity of CL-siRNA-CYP1A1 as a proof of 497 concept for targeting lung cancer, future experiments will explore different experimental 498 designs including optimizing dosage and scheduling regimen to improve efficacy.

\section{CONCLUSION}

501 This study has shown that CYP1A1 gene can be a potential target for treatment of lung

502 cancer. Cationic liposomes generated from film-coated proliposomes provided excellent

503 siRNA carriers, with subsequent ability to silence the CYP1A1 gene both in vitro and in

504 vivo. Further investigations to evaluate the aerosolization properties of CL-siRNA in animal

505 models using the proliposome approach are warranted. This study will open the doors to

506 further investigations in multiple therapeutic directions in the field of drug delivery and 507 cancer treatment.

508 
Dr. Abdelbary Elhissi would like to thank UCLan Biomedical Technology (Shenzhen) Ltd

511 for the international funding provided in collaboration with Professor Xun Sun from the

512 West China School of Pharmacy, Sichuan University (Grant UBTSL 2012-3). We also

513 thank Qatar University for providing additional funding to conduct further studies in

514 collaboration with the Anti-Doping Lab Qatar (ADLQ) (Grant QUSG-CPH-PSS-14\15-3).

515 We also thank Jianfeng Han and Chenyu Wu for their help in this present study.

\section{DECLARATION OF CONFLICTS OF INTERESTS}

517 The authors declare no conflicts of interests.

\section{REFERENCES}

Abdelrahim, M., Smith, R., 3rd, Safe, S., 2003. Aryl hydrocarbon receptor gene silencing with small inhibitory RNA differentially modulates Ah-responsiveness in MCF-7 and HepG2 cancer cells. Molecular pharmacology 63, 1373-1381. S.K., 2003. RNA interference: biology, mechanism, and applications. Microbiology and molecular biology reviews : MMBR 67, 657-685. containing propranolol hydrochloride. Journal of microencapsulation 12, 363-375. 
535 Armstrong, B., Hutchinson, E., Unwin, J., Fletcher, T., 2004. Lung cancer risk after

536 exposure to polycyclic aromatic hydrocarbons: a review and meta-analysis. Environmental 537 health perspectives 112, 970-978.

Boatright, K.M., Salvesen, G.S., 2003. Mechanisms of caspase activation. Current Opinion in Cell Biology 15, 725-731.

Brambilla, E., Gazdar, A., 2009. Pathogenesis of lung cancer signalling pathways: roadmap for therapies. The European respiratory journal 33, 1485-1497.

Bruno, R.D., Njar, V.C., 2007. Targeting cytochrome P450 enzymes: a new approach in anti-cancer drug development. Bioorganic \& medicinal chemistry 15, 5047-5060.

Buyens, K., Lucas, B., Raemdonck, K., Braeckmans, K., Vercammen, J., Hendrix, J., Engelborghs, Y., De Smedt, S.C., Sanders, N.N., 2008. A fast and sensitive method for measuring the integrity of siRNA-carrier complexes in full human serum. Journal of controlled release : official journal of the Controlled Release Society 126, 67-76.

Chen, C.M., Alli, D., 1987. Use of fluidized bed in proliposome manufacturing. Journal of pharmaceutical sciences 76,419 .

Ciolino, H.P., Yeh, G.C., 1999. The flavonoid galangin is an inhibitor of CYP1A1 activity and an agonist/antagonist of the aryl hydrocarbon receptor. British journal of cancer 79,

558 1340-1346.

Elhissi, A.M., Ahmed, W., Taylor, K.M., 2012. Laser diffraction and electron microscopy studies on inhalable liposomes generated from particulate-based proliposomes within a medical nebulizer. J. Nanosci. Nanotechnol. 12, 6693-6699.

Elhissi, A.M., Karnam, K.K., Danesh-Azari, M.R., Gill, H.S., Taylor, K.M., 2006. Formulations generated from ethanol-based proliposomes for delivery via medical

568 Fazili, I.S., Jiang, W., Wang, L., Felix, E.A., Khatlani, T., Coumoul, X., Barouki, R., 569 Moorthy, B., 2010. Persistent induction of cytochrome P4501A1 in human hepatoma cells 570 by 3-methylcholanthrene: evidence for sustained transcriptional activation of the CYP1A1 promoter. The Journal of pharmacology and experimental therapeutics 333, 99-109. 
573 Field, R.W., Withers, B.L., 2012. Occupational and environmental causes of lung cancer.

574 Clinics in chest medicine 33, 681-703.

575

576

577

578

579

580

581

582

583

584

585

586

587

588

589

590

591

592

593

594

595

596

597

598

599

600

601

602

603

604

605

606

607

608

609

610

Friedrich, J., Seidel, C., Ebner, R., Kunz-Schughart, L.A., 2009. Spheroid-based drug screen: considerations and practical approach. Nature protocols 4, 309-324.

Gala, R.P., Khan, I., Elhissi, A.M., Alhnan, M.A., 2015. A comprehensive production method of self-cryoprotected nano-liposome powders. Int. J. Pharm. 486, 153-158.

Giard, D.J., Aaronson, S.A., Todaro, G.J., Arnstein, P., Kersey, J.H., Dosik, H., Parks, W.P., 1973. In vitro cultivation of human tumors: establishment of cell lines derived from a series of solid tumors. Journal of the National Cancer Institute 51, 1417-1423.

Grit, M., Crommelin, D.J., 1993. Chemical stability of liposomes: implications for their physical stability. Chemistry and physics of lipids 64, 3-18.

Guigal, N., Seree, E., Bourgarel-Rey, V., Barra, Y., 2000. Induction of CYP1A1 by serum independent of AhR pathway. Biochemical and biophysical research communications 267, $572-576$.

Hecht, S.S., 1999. Tobacco smoke carcinogens and lung cancer. Journal of the National Cancer Institute 91, 1194-1210.

Hukkanen, J., Lassila, A., Paivarinta, K., Valanne, S., Sarpo, S., Hakkola, J., Pelkonen, O., Raunio, H., 2000. Induction and regulation of xenobiotic-metabolizing cytochrome P450s in the human A549 lung adenocarcinoma cell line. American journal of respiratory cell and molecular biology 22, 360-366.

Hwang, B.-Y., Jung, B.-H., Chung, S.-J., Lee, M.-H., Shim, C.-K., 1997. In vitro skin permeation of nicotine from proliposomes. Journal of controlled release 49, 177-184.

Jahn, A., Song, C.K., Balakrishnan, P., Hong, S.-S., Lee, J.-H., Chung, S.-J., Kim, D.-D., 2014. AAPE proliposomes for topical atopic dermatitis treatment. Journal of microencapsulation 31, 768-773.

Jung, B.H., Chung, B.C., Chung, S.-J., Lee, M.-H., Shim, C.-K., 2000a. Prolonged delivery of nicotine in rats via nasal administration of proliposomes. Journal of controlled release $66,73-79$. 
612 Jung, B.H., Chung, B.C., Chung, S.J., Lee, M.H., Shim, C.K., 2000b. Prolonged delivery of 613 nicotine in rats via nasal administration of proliposomes. Journal of controlled release : 614 official journal of the Controlled Release Society 66, 73-79.

615

616

617

618

619

620

621

622

623

624

625

626

627

628

629

630

631

632

633

634

635

636

637

638

639

640

641 642

643

644

645

646

647

Katare, O.P., Vyas, S.P., Dixit, V.K., 1990. Effervescent granule based proliposomes of ibuprofen. Journal of microencapsulation 7, 455-462.

Kellar, A., Egan, C., Morris, D., 2015. Preclinical murine models for lung cancer: Clinical trial applications. BioMed Research International, Article ID 621324, 17 pages.

Khan, I., Yousaf, S., Subramanian, S., Albed Alhnan, M., Ahmed, W., Elhissi, A., 2018. Proliposome tablets manufactured using a slurry-driven lipid-enriched powders: Development, characterization and stability evaluation. Int. J. Pharm. 538, 250-262.

Khurana, B., Goyal, A.K., Budhiraja, A., Aora, D., Vyas, S.P., 2013. Lipoplexes versus nanoparticles: pDNA/siRNA delivery. Drug delivery 20, 57-64.

Kim, H.-K., Davaa, E., Myung, C.-S., Park, J.-S., 2010a. Enhanced siRNA delivery using cationic liposomes with new polyarginine-conjugated PEG-lipid. International journal of pharmaceutics 392, 141-147.

Kim, H.K., Davaa, E., Myung, C.S., Park, J.S., 2010b. Enhanced siRNA delivery using cationic liposomes with new polyarginine-conjugated PEG-lipid. International journal of pharmaceutics 392, 141-147.

Kumar, R., Gupta, R.B., Betageri, G.V., 2001. Formulation, characterization, and in vitro release of glyburide from proliposomal beads. Drug delivery 8, 25-27.

Lorenzer, C., Dirin, M., Winkler, A.M., Baumann, V., Winkler, J., 2015. Going beyond the liver: progress and challenges of targeted delivery of siRNA therapeutics. J. Cont. Rel. 203, $1-15$.

Mercer, B.A., Lemaitre, V., Powell, C.A., D'Armiento, J., 2006. The Epithelial Cell in Lung Health and Emphysema Pathogenesis. Current Respiratory MedicineReviews 2, 101142. 
648 Mokhtar Ibrahim, M., Tawfique, S.A., Mahdy, M.M., 2013. Liposomal diltiazem $\mathrm{HCl}$ as 649 ocular drug delivery system for glaucoma. Drug development and industrial pharmacy 40 , $650 \quad 765-773$.

Murakami, A., Ashida, H., Terao, J., 2008. Multitargeted cancer prevention by quercetin. Cancer letters 269, 315-325.

Park, J.M., Ahn, B.N., Yoon, E.J., Lee, M.G., Shim, C.K., Kim, C.K., 1994. The pharmacokinetics of methotrexate after intravenous administration of methotrexate-loaded proliposomes to rats. Biopharmaceutics \& drug disposition 15, 391-407.

Payne, N.I., Browning, I., Hynes, C.A., 1986. Characterization of proliposomes. Journal of pharmaceutical sciences 75, 330-333.

Payne, N.I., Cosgrove, R.F., Green, A.P., Liu, L., 1987. In-vivo studies of amphotericin B liposomes derived from proliposomes: effect of formulation on toxicity and tissue disposition of the drug in mice. The Journal of pharmacy and pharmacology 39, 24-28.

Ruozi, B., Forni, F., Battini, R., Vandelli, M.A., 2003. Cationic liposomes for gene transfection. Journal of drug targeting 11, 407-414.

Sak, K., Everaus, H., 2015. Multi-Target Cytotoxic Actions of Flavonoids in Blood Cancer Cells. Asian Pacific journal of cancer prevention : APJCP 16, 4843-4847.

Shimada, T., Fujii-Kuriyama, Y., 2004. Metabolic activation of polycyclic aromatic hydrocarbons to carcinogens by cytochromes P450 1A1 and 1B1. Cancer science 95, 1-6. modified cationic liposomes loaded with paclitaxel and survivin siRNA for targeted

Tiwari, G., Tiwari, R., Sriwastawa, B., Bhati, L., Pandey, S., Pandey, P., Bannerjee, S.K., 2012. Drug delivery systems: An updated review. International journal of pharmaceutical investigation 2, 2-11. cancer statistics, 2012. CA: a cancer journal for clinicians 65, 87-108. 
686 Wang, J.P., Maitani, Y., Takayama, K., Nagai, T., 2000. Pharmacokinetics and antitumor

687 effect of doxorubicin carried by stealth and remote loading proliposome. Pharmaceutical 688 research 17, 782-787.

690 Whitlock, J.P., Jr., 1999. Induction of cytochrome P4501A1. Annual review of 691 pharmacology and toxicology 39, 103-125.

692

693 Zarogouldis, P., Karamanos, N.K., Porpodis, K., Domvri, K., Huang, H., Hohenforst694 Schimdt, W., Goldberg, E.P., Zarogoulidis, K., 2012. Vectors for inhaled gene therapy in 695 lung cancer. Application for nano oncology and safety of bio nanotechnology. International 696 journal of molecular sciences 13, 10828-10862.

FiGURE LEGENDS

698

699 FIGURE 1. Characterization of lipososome-siRNA complex. (a) Scanning electron

700 microscopy images of blank sorbitol and (b) Image of proliposome particles after coating

701 with the lipid. (c) Transmission electron microscopy image of cationic liposomes generated

702 from proliposomes. (d) Size and zeta potential of CL-siRAN complex at different cationic

703 liposome to siRNA ratios. (e) The mobility of siRNA complexed with cationic liposomes at

704 various molar ratios, ranging from 1-10 liposome to siRNA, by agarose gel retardation

705 assay yisualized by Goldview staining. (f) Stability of CL-siRNA complex against RNase.

706 Cationic liposomes were complexed with siRNA at different molar ratios to study the

707 degradation of siRNA by RNase by incubation with RNase at $37^{\circ} \mathrm{C}$ for up to 6 hours. The

$708 \mathrm{CL}$ and siRNA N/P ratio was kept 4:1 in all samples and siRNA alone was used as negative

709 control. 
711 FIGURE 2. Cellular uptake of CL-siRNA by A549 lung cancer cell line. (a)

712 Representative images of A549 cells transfected with FAM-siRNA, CL-FAM-siRNA or

713 Lipo2000-FAM-siRNA. Cells were treated with $5 \mu \mathrm{M}$ 3-MC to induce CYP1A1 expression

714 then incubated either with $100 \mathrm{nM}$ liposome-free FAM-siRNA or $100 \mathrm{nM}$ FAM-siRNA-

715 liposome complexes in serum-free medium. After transfection, cells were stained with 4'6-

716 diamidino-2-phenylindole (DAPI) and fluorescence images were taken by confocal

717 microscope. (b) The cellular uptake efficiency of CL-siRNA in the induced cells was also

718 measured by flow cytometry $(n=3)$.

719

720 FIGURE 3. Targeting of CYP1A1 gene using gene silencing approach. A549 lung

721 cancer cells were treated with 3-MC then transfected with CYP1A1-siRNA using liposome

722 (CL/siRNA) or Lipofectomine 2000 (Lipo2000/siRNA). Non-stimulated A549 cells were

723 used as a negative control whereas 3-MC stimulated A549 transfected with naked siRNA

724 were used as a positive control. (a) Expression of CYP1A1 gene of was analyzed by

725 quantitative RT-PCR using $\beta$-actin as internal control. Data are shown as normalized fold

726 expression relative to the untreated control $(n=3), * p<0.05$. (b) CYP1A1 enzyme activity

727 was measured by Human CYP1A1 enzyme activity fluorescence quantitative detection kits 
$728(\mathrm{n}=3), * \mathrm{p}<0.05$. (c) CYP1A1 enzyme content was detected with Human CYP1A1 ELISA

729 kits $(\mathrm{n}=3),{ }^{*} \mathrm{p}<0.05$.

731 FIGURE 4. Knockdown of CYP1A1 gene causes apoptosis in lung cancer cells. (a)

732 A549 lung cancer cells were treated with 3-MC for 24 hours then transfected with CL-

733 CYP1A1-siRNA or CYP1A1 lipofectamine-2000. Cells were then stained with flourescein-

734 conjugated annexin-V and propidium iodide (PI) and analyzed by flow cytometry.

735 Percentages of apoptotic cells are presented as mean \pm SD $(n=3)$. (b) Quantification of the

736 active caspase 3, caspase 8 and caspase 9 in 3-MC-induced A549 lung cells transfected

737 with CL- CYP1A1-siRNA as performed by Flow Cytometry using caspase activity assay

738 kits as described in the methods section. Data are presented as mean $\pm \operatorname{SD}(n=3)$.

740 FIGURE 5. Effect of CYP1A1 gene silencing on A549-mediated spheroid colonies.

741 Spheroid colonies were generated as described in the methods section. Sphere cells were

742 treated with 3-MC for $24 \mathrm{~h}$ and subsequently then transfected with CL-CYP1A1-siRNA or

743 CYP1A1 lipofectamine-2000 for $72 \mathrm{~h}$. Representative images shown are from three

744 different experiments. 
FIGURE 6. Inhibition of tumor growth using CYP1A1-siRNA in mouse model system.

747 BALB/c-nude mice were injected with 10 million A549 cells in serum free medium

748 subcutaneously into right flank. The tumor bearing mice were divided into four treatment

749 group (n=5). (a) All mice were injected with 3-MC and after 10 days were treated with (i)

750 PBS (ii) CL-CYP1A1-siRNA (iii) Naked-siRNA and (iv) Lipofectamin-complxed

751 CYP1A1-siRNA. (b) The volume of each tumor was measured at the indicated time points

752 as described in methods. Results are expressed as mean, $(n=5), \pm$ SD. (c) Mice were

753 sacrificed after 18 days with six intratumoural injection of CYP1A1 siRNA and images of

754 each tumors were taken as shown $(n=5)$. (d) Total RNA were isolated from tumor of each

755 mice. Expression of CYP1A1 gene were quantified by RT-PCR (Data expressed as mean \pm $756 \mathrm{SD} ; \mathrm{n}=3)$. 
a
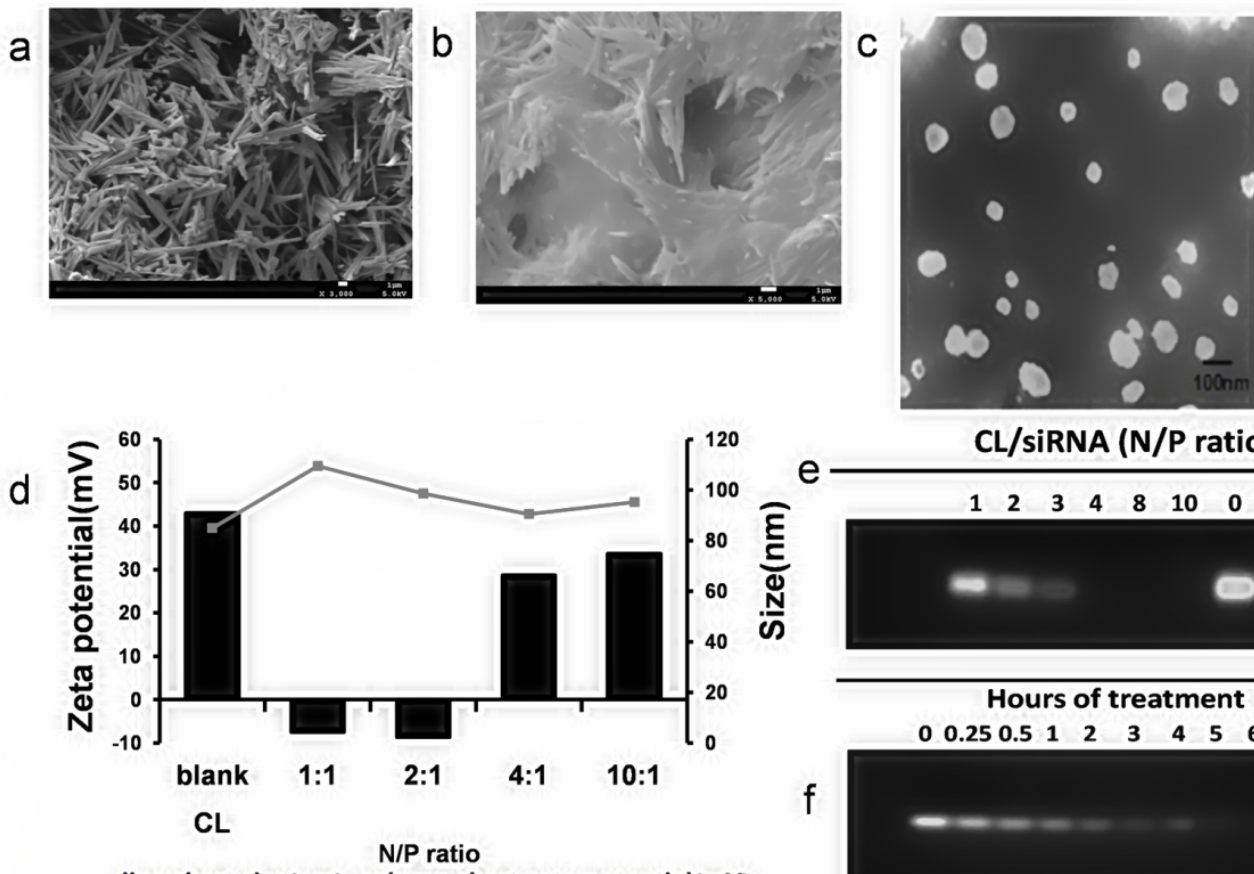

line chart:size $(\mathrm{nm})$ column chart:zeta potential $(\mathrm{mV})$

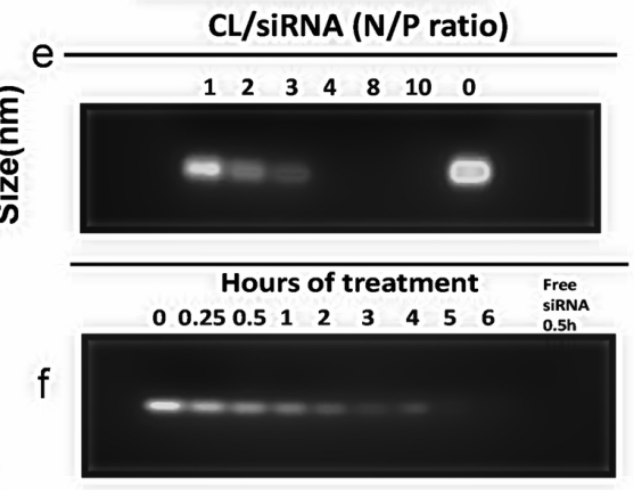



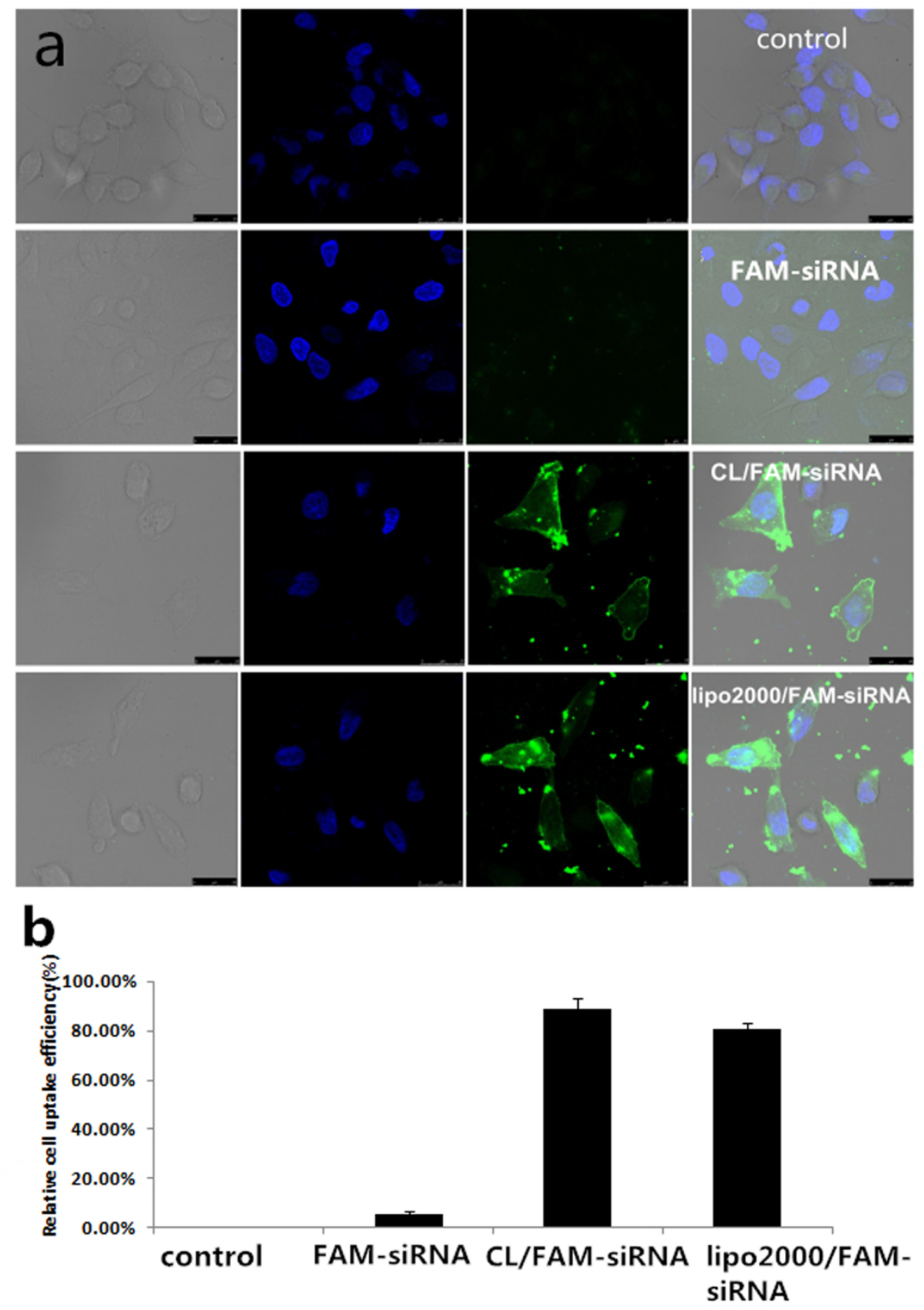


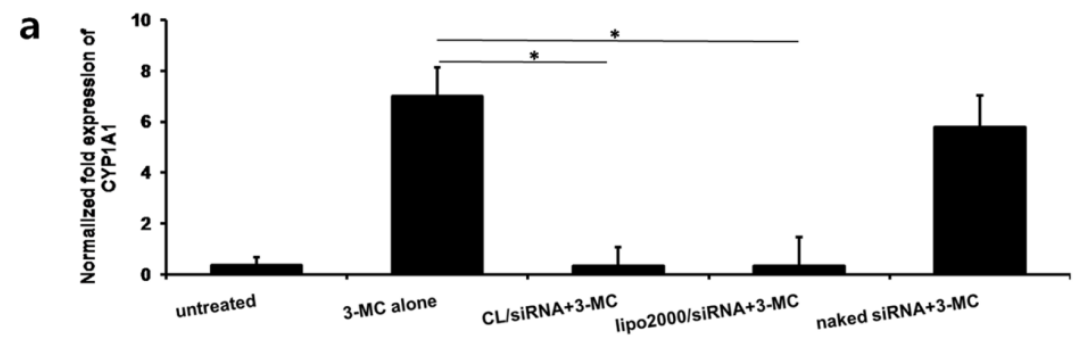

b
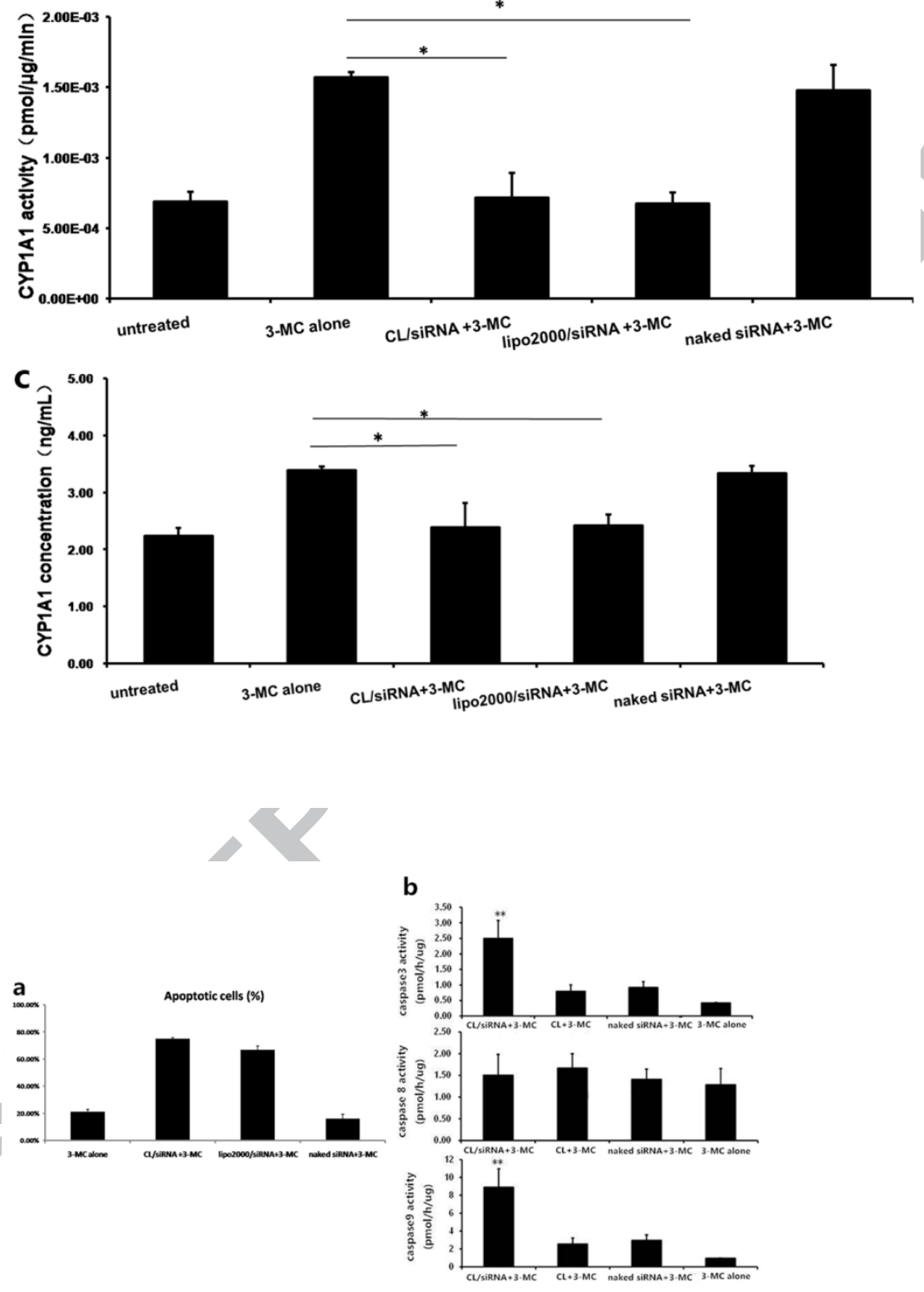


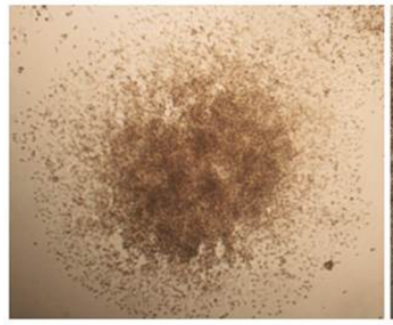

untreated

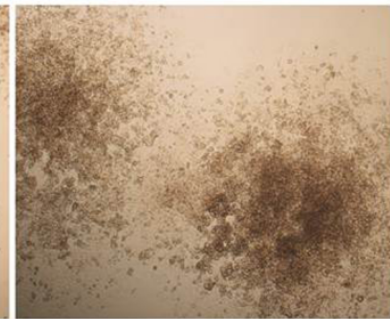

mock

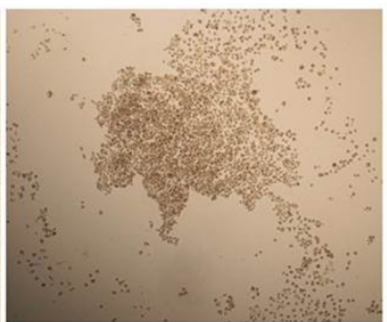

CL/SIRNA

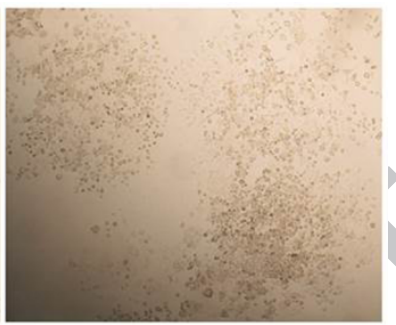

Lipo2000/siRNA
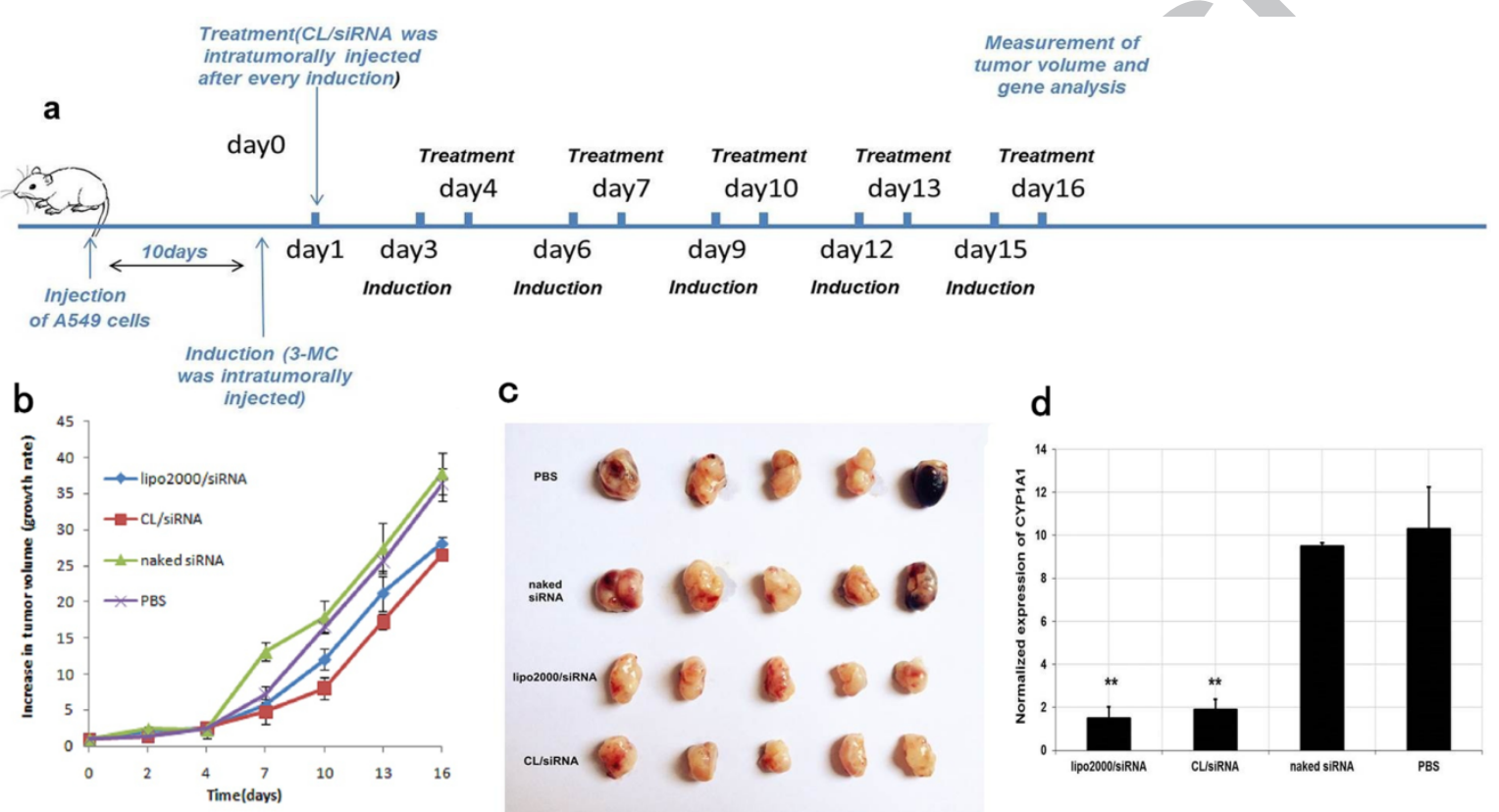

C

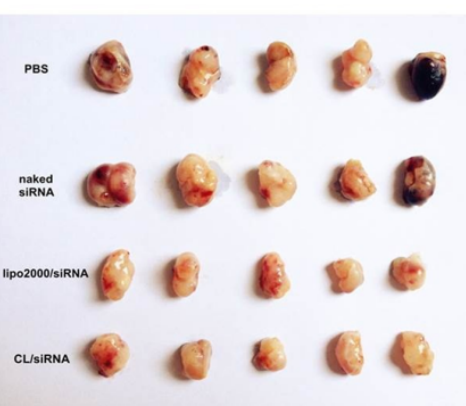

d

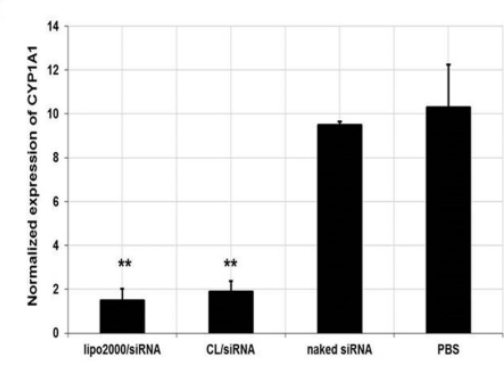

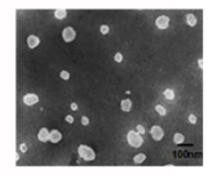
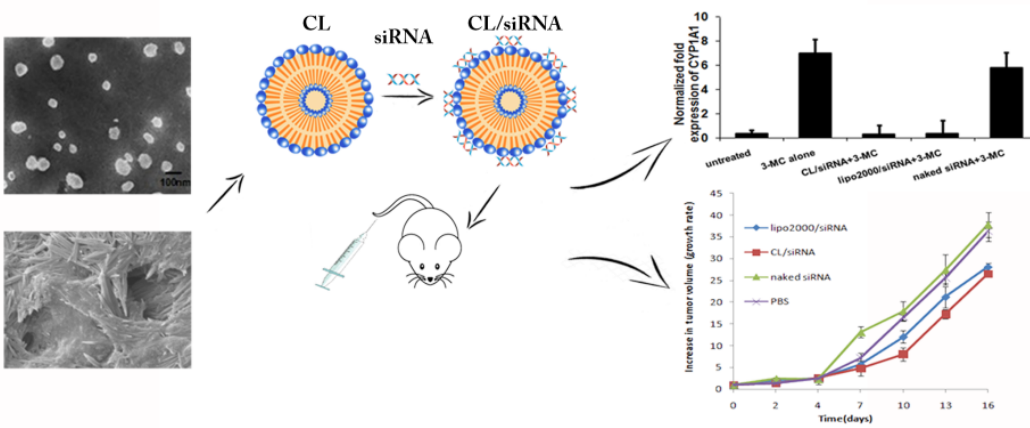

Declaration of interests

$\bigotimes$ The authors declare that they have no known competing financial interests or personal relationships that could have appeared to influence the work reported in this paper. 
768

$769 \square$ The authors declare the following financial interests/personal relationships which may be 770 considered as potential competing interests:

771

772

773

774

775

776 